\title{
Victimación delictiva en 17 países industrializados ${ }^{1}$
}

\author{
Esther Bouten, Heike Goudriaan y Paul Nieuwbeerta (iD
}

Netherlands Institute for the Study of Crime and Law Enforcement

Traducción de María José Benítez y anotaciones sobre Cataluña de Juanjo Medina

\section{Introducción}

Los resultados de la Encuesta Internacional a Víctimas del Delito confirman que los delitos contra la propiedad están disminuyendo en los países industrializados. Los mejores sistemas de seguridad en el hogar y otras formas de prevención del delito han contribuido a esta tendencia a la baja, también evidente en las estadísticas policiales. Hablando en general la Encuesta Internacional a Víctimas del Delito (EIVD) sugiere que el delito aumentó entre 1988 y 1991, no mostró mucho cambio en 1995 y descendió en 1999. Éste es el modelo dominante en muchos países industrializados. En resumen, en los diversos pases de las encuestas Nueva Zelanda, Australia y Países Bajos tienen en conjunto las tasas más altas de delincuencia, mientras que las tasas más bajas se encuentran en Japón, Irlanda del Norte y Portugal.

El presente artículo versa sobre éstas y otras importantes conclusiones fruto de la EIVD en los países industrializados y se fija en los resultados de la Encuesta Internacional a Víctimas del Delito (EIVD) de 2000. Los detalles pueden encontrarse en Van Kesteren, Mayhew y Nieuwbeerta (2000) y en Nieuwbeerta y Van Kesteren (2001).

\section{La EIVD en los países industrializados}

La EIVD es la encuesta internacional más ambiciosa conducida de forma sistemática y estandarizada (empleando una metodología común) en un gran número de países y que examina la experiencia de ciudadanos con el delito, el servicio policial, la prevención del delito y los sentimientos de inseguridad. La razón para instaurar la EIVD fue la insuficiencia de otros instrumentos, comparables internacionalmente, que midieran la naturaleza y la magnitud del delito. La cantidad de delitos registrados por la

\footnotetext{
${ }^{1}$.- Este articulo fue publicado originalmente como un capitulo en NCSR, Nieuwbeerta, P. (ed.), (2002) Crime Victimization in comparative perspective. Results from the International Crime Victims Survey, 1989-2000. Den Haag: Boom Juridische uitgevers.
}

Revista Española de Criminología

REIC NI02-03 http://www.criminologia.net

https://doi.org/10.46381/reic.v1i0.8 
policía es problemática debido a las diferencias en el modo en que la policía define, archiva y cuenta los delitos. Y puesto que la policía obtiene casi toda la información acerca de los delitos a través de los informes de las víctimas, mientras las víctimas no informen de todos los delitos, la cifra manejada por la policía puede variar de país a país en función del comportamiento de las victimas a la hora de denunciar. Es también difícil hacer comparaciones de las encuestas de victimas realizadas de forma independiente en cada país por diferentes organizaciones ${ }^{2}$, pues éstas difieren en el diseño y en el alcance. Hasta hoy las encuestas se han llevado a cabo en veinticuatro países industrializados y en cuarenta y seis ciudades en países en vías de desarrollo y en países en transición. En los países industrializados hubo cuatro pases de la EIVD, realizándose el primero en 1989, el segundo en 1992, el tercero en 1996 y el cuarto en 2000. La Tabla 1 muestra qué países industrializados han participado y en qué año, junto con los tamaños de la muestra y la media de los porcentajes de respuesta ${ }^{3}$. Puede observarse que los porcentajes de respuesta difieren considerablemente entre los países. Polonia tiene la tasa más alta de respuesta (86\%), mientras la tasa más baja de respuesta $(30 \%)$ procede de Alemania Oeste. En los países que realizaron las entrevistas cara a cara (Polonia, Irlanda del Norte y Japón), las tasas de respuesta son más altas que en los países que utilizaron la entrevista telefónica. En cada país se toma para cada pase una muestra aleatoria de hogares, regionalmente bien extendida. En el caso de los países que utilizaron entrevistas telefónicas, esto se hizo a través de diversas variantes del método de llamada telefónica al azar. En cada hogar aleatoriamente seleccionado, se entrevistaba a un sujeto de dieciséis años o más elegido también al azar.

\footnotetext{
${ }^{2}$.- Nota del traductor: Por ejemplo, las encuestas del Ministerio del Interior español con la British Crime Survey.

${ }^{3}$.- Nota del traductor: España como puede observarse solo participó a nivel nacional en el pase de 1989. Para mayor colmo de males, la ridículamente e inexplicable baja tasa de respuesta limita de forma considerable los datos obtenidos en dicho pase. En España, aunque el Ministerio de Interior, comisiona la administración de encuestas al CIS con cuestionarios diseñados bien por la Policía Nacional, bien por la Guardia Civil, no ha existido el interés político para financiar la participación de España en la EIVD. Ello a pesar de que la calidad científica de los cuestionarios del Ministerio de Interior a la hora de medir victimaciones es considerada como muy pobre por parte de la comunidad criminólogica española. Más recientemente, la Comunidad Autónoma de Cataluña decidió de forma laudable participar en este programa de investigación y financió el pase en el 2000 de la EIVD.
}

Revista Española de Criminología

REIC NI02-03 http://www.criminologia.net 
Tabla 1. EIVD. Encuestas nacionales en países industrializados: tamaños de la muestra $y$ media de los porcentajes de respuesta.

\begin{tabular}{|c|c|c|c|c|c|}
\hline País & 1989 & 1992 & 1996 & 2000 & Media del índice de respuesta $(\%)$ \\
\hline Australia & 2,012 & 2,006 & ---- & 2,402 & 53 \\
\hline Austria & --- & ---- & 1,507 & ---- & 76 \\
\hline Bélgica & 2,060 & 1,485 & ---- & 2,402 & 45 \\
\hline Canadá & 2,074 & 2,152 & 2,134 & 2,078 & 58 \\
\hline Cataluña (España) & ---- & ---- & ---- & 2,909 & 73 \\
\hline Dinamarca & ---- & ---- & ---- & 3,007 & 66 \\
\hline Inglaterra y Gales & 2,006 & 2,001 & 2,171 & 1,947 & 48 \\
\hline Finlandia & 1,025 & 1,620 & 3,899 & 1,783 & 82 \\
\hline Francia & 1,502 & ---- & 1,003 & 1,000 & 52 \\
\hline Alemania (Oeste) & 5,274 & ---- & ---- & ---- & 30 \\
\hline Italia & --- & 2,024 & ---- & ---- & 61 \\
\hline Japón (a) & 2,411 & 2,382 & ---- & 2,211 & 78 \\
\hline Países Bajos & 2,000 & 2,000 & 2,008 & 2,001 & 63 \\
\hline Nueva Zelanda & ---- & 2,048 & ---- & ---- & 65 \\
\hline Irlanda del Norte & 2,000 & ---- & 1,042 & 1,565 & 82 (b) \\
\hline Noruega & 1,009 & ---- & ---- & ---- & 71 \\
\hline Polonia (c) & ---- & 2,033 & 3,483 & 5,276 & 86 \\
\hline Portugal & ---- & --- & ---- & 2,000 & 56 \\
\hline Escocia & 2,007 & ---- & 2,194 & 2,040 & 52 \\
\hline España & 862 & ---- & --- & ---- & 33 \\
\hline Suecia & ---- & 1,707 & 1,000 & 2,000 & 72 \\
\hline Suiza & 1,000 & --- & 1,000 & 4,234 & 64 \\
\hline E.E.U.U. & 1,996 & 1,501 & 1,003 & 1,000 & 44 \\
\hline Total & 29,328 & 22,959 & 20,937 & 39,458 & $61(d)$ \\
\hline
\end{tabular}

(a).- No todos los datos están disponibles para Japón.

(b).- Media en 1996 y 2000; el índice de respuesta en 1989 no se conoce.

(c).- Los resultados de Polonia no se publicaron en los informes de la tercera EIVD en los países industrializados.

(d).- El 50\% cuando se controla para las diferencias en los tamaños de la muestra de los distintos países.

\section{Tasas de victimación.}

En las EIVD a los sujetos seleccionados se les preguntaba si habían sido víctimas en los últimos cinco años de una de las ocho formas de delito contra la propiedad (robo de coche, robo en el coche ${ }^{4}$, vandalismo hacia el coche, robo de motocicleta, robo de bicicleta, robo dentro de la casa, tentativa de robo en el hogar, robo de objetos (bienes) personales) y/o de una de las tres formas de delito contra las personas $^{5}$ (atraco, incidentes sexuales, agresiones y amenazas). Esta encuesta es parecida a la mayoría de las encuestas de victimación con relación a los delitos que

${ }^{4}$.- Nota del traductor: Robo del coche se refiere a la sustracción del vehículo, mientras que robo en el coche se refiere a la sustracción de objetos de dentro o fuera del mismo (radio, guantera, retrovisores, ruedas, etc.).

${ }^{5}$.- Nota del traductor: Nótese que incluimos como delito contra la persona a los atracos, aunque en el Código Penal español este es considerado como un delito contra la propiedad. En términos criminológicos el carácter violento de la confrontación entre víctima y agresor hace que a menudo se clasifique juntamente con otros delitos violentos contra las personas con una motivación instrumental diferente.

Revista Española de Criminología

REIC NI02-03 http://www.criminologia.net 
comprende. Se limita a contar delitos contra sujetos claramente identificables, excepto los niños. (Las encuestas de víctimas del delito no pueden abarcar fácilmente a las víctimas de delitos de cuello blanco o de delitos sin víctima tales como las del abuso de drogas).

Figura 1. Victimación general

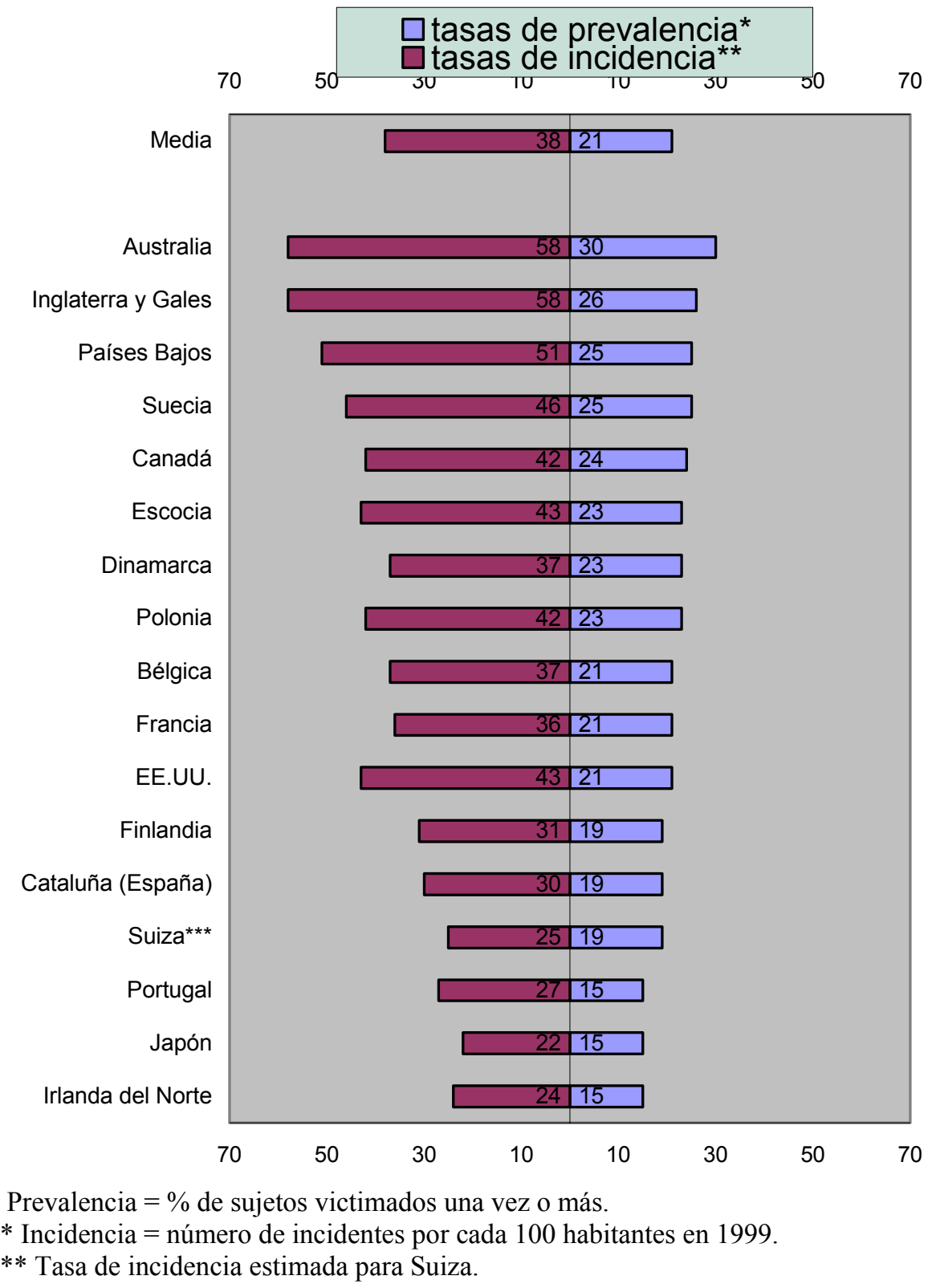

Para los delitos que abarca, la EIVD pregunta sobre incidentes en general de acuerdo con la definición legal de los delitos. En esencia, acepta sin cuestionar la descripción de los hechos proporcionada por los sujetos seleccionados $-\mathrm{O}$ al menos los informes que están preparados para dar a los entrevistadores. A este respecto la encuesta emplea una definición más amplia de delito que la policía -quien si recibe los 
incidentes, probablemente no registra aquellos que se consideran no merecedores de la atención del sistema de justicia penal o que no reúnen los requisitos establecidos por la organización en cuanto a lo que se considera evidencia razonable de la ocurrencia de un delito.

La Figura 1 muestra las tasas de incidencia (el número de delitos sufridos por cada 100 personas de la muestra), así como las tasas de prevalencia (porcentaje de sujetos seleccionados que sufrieron un delito específico una o más veces) en el año anterior a 2000. La encuesta calculó el término medio de victimación sobre once formas de delito en los diecisiete países industrializados que tomaron parte en la EIVD de 2000. Cuando observamos los once tipos de delitos, el porcentaje de sujetos que son victimados una o más veces en el último año (en el año anterior a la encuesta) (tasas de prevalencia) y también el número de delitos por cada cien habitantes (tasas de incidencia) parece ser más elevado en Australia, Inglaterra y Gales y Países Bajos (tasas de incidencia $>-51 \%$ e índices de prevalencia $>-25 \%$ ). Japón e Irlanda del Norte tienen, en general, las tasas de prevalencia e incidencia más bajos (tasas de incidencia $<-24 \%$ y tasas de prevalencia $=15 \%)^{6}$.

Tabla 2. El perfil del delito en diferentes países (porcentaje de todos los delitos: total $=100 \%$ ): EIVD 2000 (a)

\begin{tabular}{|c|c|c|c|c|c|c|}
\hline País & $\begin{array}{l}\text { Robos en y } \\
\text { de coches }\end{array}$ & $\begin{array}{c}\text { Actos } \\
\text { vandálicos } \\
\text { contra } \\
\text { coches }\end{array}$ & $\begin{array}{c}\text { Robo de } \\
\text { motocicleta } \\
\text { y de } \\
\text { bicicleta }\end{array}$ & $\begin{array}{l}\text { Robo dentro } \\
\text { del hogar } \\
\text { (consumados } \\
\text { y tentativas) }\end{array}$ & $\begin{array}{c}\text { Todos los } \\
\text { delitos } \\
\text { contra las } \\
\text { personas (b) }\end{array}$ & $\begin{array}{c}\text { Robo de } \\
\text { objetos } \\
\text { personales }\end{array}$ \\
\hline Australia & 18 & 20 & 4 & 15 & 29 & 14 \\
\hline Bélgica & 14 & 23 & 15 & 17 & 19 & 13 \\
\hline Canadá & 20 & 15 & 10 & 13 & 27 & 15 \\
\hline $\begin{array}{l}\text { Cataluña } \\
\text { (España) }\end{array}$ & 25 & 38 & 4 & 7 & 14 & 12 \\
\hline Dinamarca & 14 & 13 & 26 & 13 & 21 & 12 \\
\hline $\begin{array}{l}\text { Inglaterra y } \\
\text { Gales }\end{array}$ & 19 & 23 & 6 & 12 & 30 & 10 \\
\hline Finlandia & 11 & 16 & 19 & 6 & 36 & 13 \\
\hline Francia & 23 & 31 & 6 & 8 & 24 & 9 \\
\hline Japón & 8 & 26 & 40 & 13 & 11 & 2 \\
\hline Países Bajos & 12 & 26 & 21 & 10 & 19 & 12 \\
\hline $\begin{array}{l}\text { Irlanda del } \\
\text { Norte }\end{array}$ & 20 & 25 & 10 & 11 & 22 & 11 \\
\hline Polonia & 21 & 23 & 10 & 10 & 20 & 16 \\
\hline Portugal & 29 & 33 & 4 & 13 & 14 & 8 \\
\hline Escocia & 16 & 30 & 6 & 9 & 28 & 12 \\
\hline Suecia & 19 & 14 & 21 & 7 & 24 & 16 \\
\hline E.E.U.U. & 20 & 22 & 7 & 15 & 20 & 16 \\
\hline Total & 18 & 24 & 13 & 11 & 22 & 12 \\
\hline
\end{tabular}

(a).- Basado en tasas de incidencia. Porcentajes de delitos por país suman al 100\%. (b).- Basado en atraco, incidentes sexuales y agresiones y amenazas.

${ }^{6}$ - Nota del traductor: Nótese que Cataluña presenta tanto una incidencia como una prevalencia bastante baja, si la misma es comparada con el resto de los países industrializados.

Revista Española de Criminología

REIC NI02-03 http://www.criminologia.net 
En países donde la incidencia es alta respecto a la prevalencia, hay más concentración del delito entre aquellos que son victimados. En Nueva Zelanda, Estados Unidos, España y Australia la disparidad es más amplia, sugiriendo que cuando las personas son víctimas éstas son más propensas a la victimación repetida. En Japón y Cataluña, por otro lado, la disparidad entre las tasas de prevalencia e incidencia es la menor, sugiriendo incluso una distribución menos concentrada.

El perfil del delito en los diferentes países reflejará el modelo de riesgo de victimación y la frecuencia de un tipo de victimación con relación a otra. No se dice mucho respecto a los niveles relativos de victimación, pero es un modo útil de mostrar cómo el peso del delito en términos cuantitativos difiere entre los diversos países. La Tabla 2 muestra los principales patrones para la EIVD de $2000^{7}$. El análisis se basa en tasas de incidencia, con el número total de delitos fijados al 100\%.

Puede verse que los delitos contra las personas abarcan cerca de la cuarta parte de todos los delitos, con agresiones y amenazas constituyendo cerca de las dos terceras partes de éstos (o el 15\% de todos los delitos). Las formas de atraco representan una proporción mínima de los delitos contra las personas y esto es válido para todos los países. Los actos vandálicos contra coches también constituyen cerca de la cuarta parte de incidentes sufridos en general. El robo de y en coches considerados conjuntamente comprende algo menos de una quinta parte de todos los delitos (18\%), teniendo los robos en coches una representación mayor (15\%). La mayor diferencia entre países es respecto al robo de bicicletas, que refleja los diversos porcentajes de propiedad de las mismas. En resumen el perfil del delito de acuerdo con la EIVD de 2000 es muy parecido al resultante de los pases previos de la EIVD, cuando fueron comparados los países que tomaron parte ${ }^{8}$.

\section{Gravedad del delito}

Para tener en cuenta las diferencias en la gravedad de los delitos, se introdujo una pregunta en la EIVD de 1992 que fue posteriormente repetida en el pase del 2000,

\footnotetext{
${ }^{7}$.- Suiza se omite, porque los índices de incidencia de 2000 no están disponibles para valorar su perfil nacional.

${ }^{8}$.- Nota del Traductor: Nótese que en Cataluña los delitos contra vehículos representan un porcentaje muy elevado del total de delitos detectados por la encuesta. De hecho, Cataluña representa el país con el mayor porcentaje de vandalismo contra vehículos en relación con el total de la criminalidad. De forma igualmente notable, y consistente con los bajos niveles de incidencia y prevalencia del delito, en Cataluña los delitos de tipo violento contra las personas representan un porcentaje particularmente bajo del total de la criminalidad.
}

Revista Española de Criminología

REIC NI02-03 http://www.criminologia.net 
pidiendo a las víctimas que valorasen la gravedad de lo ocurrido. La Tabla 3 muestra qué proporción de delitos de la EIVD fueron considerados muy graves, medianamente graves y no muy graves, de acuerdo con las víctimas de diecisiete países industrializados en la EIVD de 2000. Ciertos delitos se subdividen (por ejemplo los robos de coches se dividen dependiendo de si el coche fue recuperado o no).

Los robos de coches en los que el propietario no consiguió recuperar el coche fueron considerados los más graves (el $57 \%$ de los incidentes fueron percibidos como muy graves). Los siguientes delitos considerados más graves fueron las agresiones sexuales (54\%) y luego los robos de coche si el coche fue recuperado (46\%). La gravedad otorgada al robo de coche es probablemente un reflejo tanto de la incomodidad de tener el coche robado como de la gran cantidad de dinero que generalmente supone la pérdida, incluso aunque pueda haber compensación por parte del seguro. El atraco portando arma (implicando el uso de arma) (el 45\% de los incidentes se consideraron muy graves) se estimó prácticamente en el mismo nivel que los robos de coches recuperados. Las agresiones con violencia (41\%) fueron equivalentes a los allanamientos de morada en los que alguien entró en el hogar para robar. Los delitos menos graves fueron los actos vandálicos contra coches (sólo un 13\% fueron considerados muy graves), el robo en coches (14\%) y el robo de bicicleta (15\%).

Tabla 3. Gravedad de los delitos según las víctimas de 17 países en la EIVD de 2000

\begin{tabular}{lccc}
\hline \multicolumn{2}{c}{ Muy grave (\%) } & Bastante grave (\%) & No muy grave (\%) \\
\hline Robo de coche: no recuperado & 57 & 31 & 11 \\
Agresiones sexuales & 54 & 30 & 16 \\
Robo de coche: recuperado & 46 & 38 & 17 \\
Atraco con arma & 45 & 34 & 21 \\
Agresión & 41 & 34 & 25 \\
Robo en el hogar & 40 & 34 & 25 \\
Robo de una motocicleta & 0 & 40 & 26 \\
ciclomotor & 34 & 38 & 30 \\
Atraco sin arma & 32 & 41 & 32 \\
Otro robo de objetos personales & 27 & 37 & 36 \\
Amenazas & 27 & 30 & 49 \\
Comportamiento sexual ofensivo & 21 & 31 & 45 \\
Intento de robo en el hogar & 20 & 35 & 48 \\
Robo de la cartera & 20 & 37 & 52 \\
Robo de bicicleta & 15 & 34 & 56 \\
Robo en el coche & 14 & 31 & \\
\hline Acto vandálico contra el coche & 13 & & \\
\hline
\end{tabular}

Otra cuestión es si las valoraciones de gravedad para los diferentes tipos de delitos varían de unos países a otros de tal modo que podrían sugerir diferentes umbrales de tolerancia o actitud frente a los diversos delitos. La posición relativa de la gravedad de los once tipos de delitos difiere poco entre los países. Los sujetos de los

Revista Española de Criminología

REIC NI02-03 http://www.criminologia.net 
distintos países juzgaron la relativa gravedad de los diferentes delitos con un grado de consistencia considerable, de nuevo sugiriendo un amplio consenso acerca del valor del delito convencional. En resumen, el robo de coche fue estimado como el primero, segundo o tercer delito más grave en todos los países, excepto en Dinamarca. El robo dentro del hogar estuvo dentro de los tres delitos más graves en todos los países, excepto en Japón e Irlanda del Norte. El atraco estuvo entre los tres delitos más graves en los once países. En Dinamarca las agresiones y amenazas e incidentes sexuales fueron estimados como más graves que el robo de coche; en Polonia el robo de motocicleta fue valorado como más grave que el robo en casa y el atraco; en Portugal las agresiones y amenazas y los incidentes sexuales fueron considerados como más graves que el robo en el hogar; y las víctima en Japón estimaron el robo de objetos personales y las agresiones y amenazas como delitos más serios que el robo en el hogar y el atraco. Además, el acto vandálico contra coche fue considerado como el delito menos serio en general. Recibió la valoración más baja en todos los países, excepto en Dinamarca. Finalmente, en trece países, el robo en el coche, el robo de bicicleta y el acto vandálico contra coche estuvieron entre las valoraciones más bajas.

En conjunto los sujetos de Irlanda del Norte, Cataluña, Inglaterra y Gales y Japón dieron los valores de gravedad más altos para los once delitos, mientras que la clasificaciones medias en Dinamarca, Finlandia y Países Bajos fueron las más bajas. Estas diferencias podrían reflejar las posibles diferencias en la naturaleza del delito sufrido (que no son particularmente bien medidas en la EIVD) -o algún grado de variación en actitudes hacia el delito. Ello podría también deberse a las diferencias en la connotación y traducción de la palabra "grave".

\section{Las víctimas y la policía}

\subsection{Denuncias ante la Policía}

Hay diferencias en las tasas de denuncias para los diferentes tipos de delitos. En la mayoría de los países, casi todos los coches y motocicletas robados se denunciaron, así como la mayoría de los robos dentro del hogar. Cerca de dos terceras partes de los robos en coches se comunicaron a la policía y más de la mitad de los robos de bicicleta y atracos. Solamente sobre una tercera parte de todos las agresiones y amenazas se denunciaron a la Policía; la cifra fue más elevada para las agresiones con violencia (45\%) que para las amenazas (29\%). Los incidentes sexuales se denunciaron menos

Revista Española de Criminología

REIC NI02-03 http://www.criminologia.net 
frecuentemente (por término medio el 15\%). El veintiocho por ciento de los incidentes sexuales se denunciaron cuando los encuestados manifestaron que habían sido agredidos/as sexualmente, pero cuando sólo había tenido lugar un comportamiento ofensivo de naturaleza sexual, los incidentes denunciados a la Policía fueron el diez por ciento

Para valorar las propensiones relativas a denunciar el delito en los diferentes países, la proporción global de todos los delitos denunciados no es una medida razonable. Esto es así porque los niveles de denuncia varían según el tipo de delito, de este modo en general los niveles de denuncia estarán influidos por la constitución del delito en cada país. La Figura 2 ofrece una solución intermedia al mostrar los niveles de denuncia medios sobre seis tipos de delitos para los que los niveles de denuncia son los más variables y/o la experiencia de victimación es comparativamente alta ${ }^{9}$. Puede observarse que Dinamarca, Suecia, Irlanda del Norte y Países Bajos tienen las tasas de denuncia más elevados, con al menos el $58 \%$ de los delitos denunciados. Las tasas de denuncia más bajos se encuentran en Portugal, Japón, Cataluña y Polonia, donde se denuncia cerca de uno de cada tres delitos.

Figura 2. Porcentaje de delitos denunciados a la Policía según las víctimas en los 17 países que tomaron parte en la EIVD de 2000

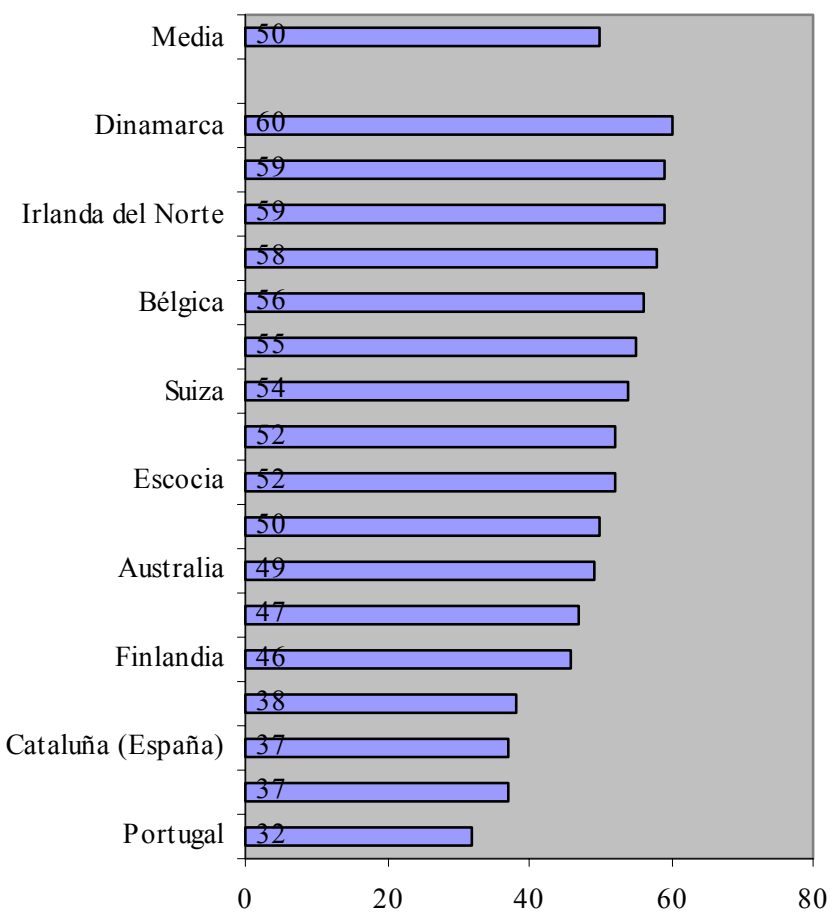

\footnotetext{
9.- Los índices (porcentajes) de denuncia relativos al último incidente (delito) sufrido durante los últimos cinco años, para robo en coches, actos vandálicos contra coches, robo de bicicleta, robo dentro del hogar, intento de robo dentro del hogar y robos de objetos personales.
}

Revista Española de Criminología

REIC NI02-03 http://www.criminologia.net 


\subsection{Satisfacción de las víctimas con relación a la Policía}

En los pases de la EIVD de 1996 y 2000, a las víctimas que habían denunciado a la Policía se les preguntó si estaban satisfechas con la respuesta policial ${ }^{10}$. La Figura 3 muestra el porcentaje de víctimas que estuvieron satisfechas en 2000, para los dos delitos contra la propiedad y para los tres delitos contra las personas considerados conjuntamente.

En ambos pases, no hay muchas diferencias entre cómo se sintieron las víctimas cuando denunciaron delitos contra la propiedad y cuando denunciaron delitos contra las personas. Para ambos, cerca de seis de cada diez encuestados se sintieron satisfechos como promedio. Sin embargo, cuando se observan los países individualmente considerados, las víctimas estuvieron a menudo menos satisfechas con la asistencia policial en los delitos contra las personas (esto ocurrió en nueve países), que con la asistencia policial en los delitos contra la propiedad (cinco países). En el resto de países hubo poca diferencia ${ }^{11}$. Además, los niveles de satisfacción con la Policía después de denunciar fueron más bajos cuando los delitos fueron evaluados como graves que cuando fueron juzgados "algo graves" o "no muy graves". A primera vista esto podría sugerir que la Policía hace un trabajo de más baja calidad cuando se denuncian delitos "graves". Sin embargo, parece más probable que las víctimas tengan mayores expectativas de la Policía cuando denuncian delitos que consideran como más graves.

\footnotetext{
${ }^{10}$ - Las víctimas de robos de coches, robos dentro del hogar (robo con allanamiento de morada), atraco, incidentes sexuales y agresiones y amenazas solamente.

${ }^{11}$.- Nota del traductor: Nótese que los niveles de satisfacción con la policía en Cataluña son de los más elevados entre todos los países participantes en la EIVD. Esto es consistente con los indicadores sobre satisfacción con la policía generados por el CIS. En todo caso, ha de notarse que el hecho de que sean tan pocos los delitos que se denuncian en España puede de alguna forma estar jugando un papel en el nivel de satisfacción. Por un lado, las personas valorando el nivel de satisfacción es menor, lo cual crea medidas de satisfacción mas inestables. Por otra parte, puede en la medida que personas con una mayor satisfacción con la policía sean más predispuestas a denunciar, esta valoración sobre satisfacción puede ser un resultado de auto-selección de la muestra. Y, finalmente, niveles relativamente bajos de denuncia también significan una menor presión en las fuerzas policiales que, como consecuencia, deberían estar en mejores condiciones para prestar un servicio más atento.
}

Revista Española de Criminología

REIC NI02-03 http://www.criminologia.net 
Figura 3. Porcentaje de sujetos satisfechos con la respuesta policial después de la denuncia*

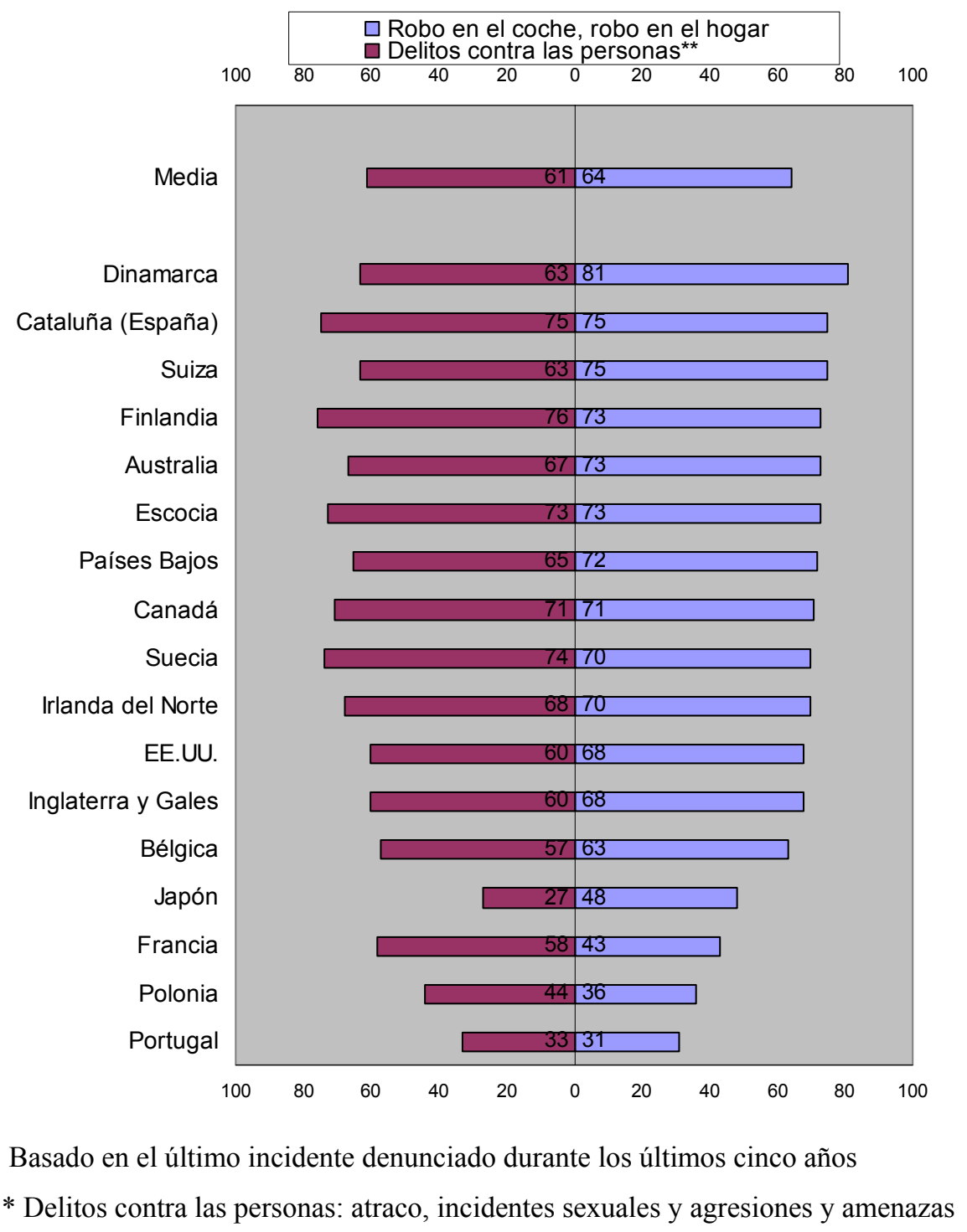

A los encuestados que indicaron que no estaban satisfechos con la forma en que les trató la Policía se les preguntó por qué no estaban satisfechos. Las principales causas de insatisfacción fueron que la Policía "no hizo lo suficiente" y que "no estaba interesada". Algunas otras razones para explicar la insatisfacción fueron que el ofensor no había sido cogido, la falta de cortesía o mala educación de la Policía (sobre todo de importancia para las víctimas de agresiones y amenazas), no recuperar ninguno de los objetos robados (sobre todo para víctimas de robo en coche y robo dentro del hogar) y falta de información por parte de la Policía (sobre todo para víctimas de incidentes sexuales). 


\subsection{Ayuda a la víctima}

En la EIVD, a las víctimas de robo dentro del hogar y delitos contra las personas (atraco, incidentes sexuales o agresiones y amenazas), y que denunciaron esto a la Policía, se les preguntó si habían recibido ayuda desde una oficina especializada de apoyo a la víctima. A aquéllos que no habían recibido ninguna ayuda se les preguntó si habrían agradecido ayuda para obtener información o apoyo práctico o emocional. Los resultados de 2000 están en la Tabla 4.

Tabla 4. Porcentaje de víctimas que recibieron ayuda o que habrian agradecido recibir ayuda de una oficina especializada (EIVD2000) (a)

\begin{tabular}{lcccc}
\hline & \multicolumn{2}{c}{ Ayuda recibida (b) } & \multicolumn{2}{c}{ La ayuda habría sido útil (c) } \\
\hline & $\begin{array}{c}\text { Robo en el } \\
\text { hogar }\end{array}$ & $\begin{array}{c}\text { Delito contra las } \\
\text { personas (d) }\end{array}$ & Robo en el hogar & $\begin{array}{c}\text { Delito contra las } \\
\text { personas (d) }\end{array}$ \\
Australia & 3 & 7 & 21 & 33 \\
Bélgica & 3 & 7 & 22 & 34 \\
Canadá & 3 & 22 & 30 & 31 \\
Cataluña (España) & 7 & 3 & 49 & 59 \\
Dinamarca & 1 & 19 & 26 & 40 \\
Inglaterra y Gales & 16 & 20 & 29 & 36 \\
Finlandia & -- & 2 & 35 & 35 \\
Francia & -- & 3 & 14 & 24 \\
Japón & -- & -- & 39 & 48 \\
Países Bajos & 9 & 16 & 13 & 23 \\
Irlanda del Norte & 18 & 23 & 41 & 45 \\
Polonia & na & 4 & na & 51 \\
Portugal & -- & -- & 52 & 48 \\
Escocia & 12 & 12 & 36 & 35 \\
Suecia & 5 & 16 & 29 & 29 \\
EE.UU. & 5 & 12 & 33 & 38 \\
\hline Todos los países (e) & 5 & 10 & 31 & 41 \\
\hline
\end{tabular}

(a).- No se dispone de los resultados de Suiza; ni de los resultados para el robo en Polonia.

(b).- Sólo las víctimas que informaron a la Policía.

(c).- Sólo las víctimas que informaron a la Policía y no recibieron ayuda.

(d).- Basados en atraco, incidentes sexuales y agresiones y amenazas.

(e).- Los datos de Polonia fueron omitidos a la hora de calcular la media entre todos los países

Por término medio en 2000, la atención es recibida más frecuentemente por las víctimas de delitos contra las personas que por las víctimas de robo dentro del hogar (el $10 \%$ recibió ayuda, contra el 5\% de víctimas de robo en el hogar). Las víctimas en Reino Unido -donde el movimiento de asistencia a víctimas está consolidado- a menudo la mayoría recibieron apoyo, relativamente con poca diferencia entre víctimas de robo en el hogar y víctimas de delitos contra las personas. Poco o ningún apoyo parece haber sido recibido en Portugal, Japón, Finlandia, Francia y Polonia. Comparando las cifras de la EIVD de 2000 con aquéllas de 1996, hubo poco cambio en 
la proporción de víctimas contactadas por las oficinas de apoyo después de que ellas denunciaran los hechos a la Policía.

En general, cerca de una de cada tres víctimas de robo dentro del hogar y cerca de cuatro de cada diez víctimas de delitos contra las personas que no recibieron ninguna ayuda por parte de una oficina de apoyo asistencia consideraron que habría sido útil si hubieran recibido ayuda. La mayor necesidad fue expresada por los sujetos de Cataluña $^{12}$, Polonia, Portugal, Japón e Irlanda del Norte (a pesar del relativamente alto nivel de ayuda actualmente ofrecido en este último país).

Figura 4. Porcentaje de víctimas que piensan que la Policía es servicial y hace un buen trabajo para controlar el delito en su zona

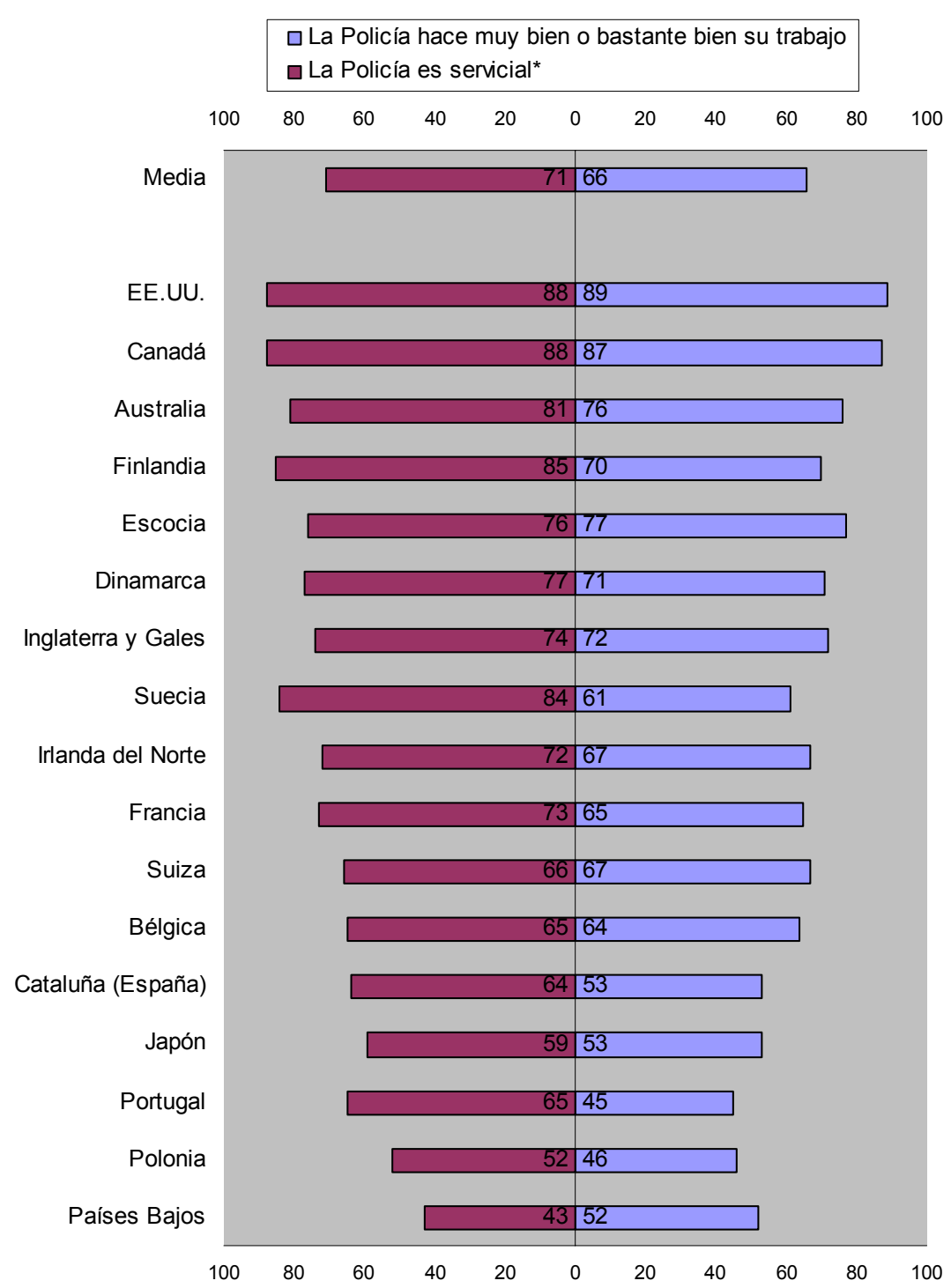

* Basado en aquéllos que están completamente de acuerdo y tienden a estar de acuerdo en que la Policía es servicial.

\footnotetext{
${ }^{12}$ - Nota del Traductor: Nótese que Cataluña es uno de los países con una menor prevalencia de asistencia a las víctimas de delitos contra las personas de tipo violento.

Revista Española de Criminología

REIC NI02-03 http://www.criminologia.net
} 


\section{Actitudes generales hacia la policía}

Con excepción de la opinión de las víctimas hacia la Policía, a todos los sujetos seleccionados se les pidió que emitieran un juicio sobre la actuación en general de la Policía. Generalmente, en aquéllos países donde la gente piensa que la Policía hace un buen trabajo controlando el delito, la Policía también se considera eficaz y viceversa. Los más satisfechos fueron los sujetos de Estados Unidos y Canadá, donde cerca de nueve de cada diez sujetos opinaron que la Policía actuaba bien y que era eficaz. Las valoraciones más negativas de la actuación policial se dieron en Portugal, Polonia y Países Bajos (ver Figura 4). La Policía fue considerada relativamente ineficaz en estos países también, aunque no especialmente así en Portugal. Y aunque las diferencias entre países son relativamente grandes, las causas de estas diferencias no están claras ${ }^{13}$.

\section{Actitudes hacia el delito}

\subsection{Probabilidad estimada de robo dentro del hogar}

La EIVD proporciona una medida que tiene que ver con lo preocupado que los encuestados están con el robo dentro del hogar a través de una pregunta que trata de estimar la probabilidad que existe de que en el próximo año sus hogares sean objeto de robo. La Figura 5 muestra el porcentaje de sujetos que estimaron la posibilidad de robo como "muy probable" o "probable". Los sujetos de Portugal (58\%), Bélgica y Francia (cerca del 45\%) fueron los más pesimistas. Hubo menos preocupación en los Países Escandinavos, Estados Unidos y Países Bajos (20\% o menos).

\footnotetext{
${ }^{13}$.- Nota del traductor: Nótese que aunque en Cataluña, los niveles de satisfacción con la policía una vez se denuncia un delito son elevados. El nivel general de satisfacción con la eficacia de la labor policial no es particularmente elevado y de hecho se encuentra entre los países con niveles más bajos de satisfacción con dicha labor policial. Ello sería un factor que contribuiría a explicar la baja tasa de denuncias de los delitos sufridos.
}

Revista Española de Criminología

REIC NI02-03 http://www.criminologia.net 
Figura 5. Porcentaje de robo en el hogar considerado probable o muy probable en el año próximo

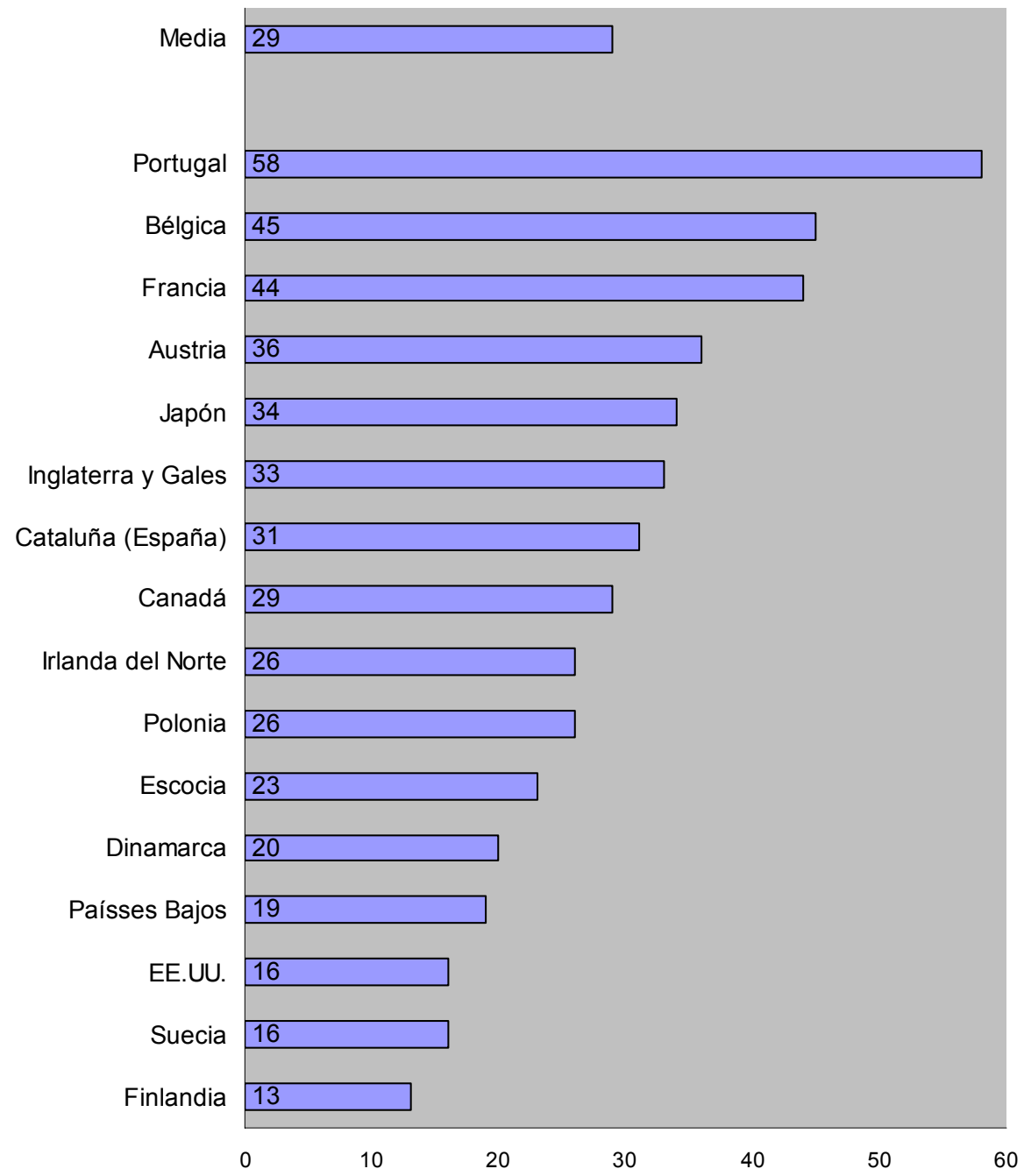

La EIVD ha encontrado previamente que las percepciones de la probabilidad de robo en el nivel nacional están fuertemente relacionadas con los riesgos de robo de las EIVD nacionales, por ejemplo, los países donde se sienten más vulnerables al robo (en las proporciones más altas) en el próximo año son aquéllos donde los riesgos son efectivamente más elevados ${ }^{14}$. En el nivel individual, los resultados de la EIVD están en línea con una colección importante de datos proveniente de las encuestas a víctimas llevadas a cabo localmente en los diferentes países que considera que la experiencia personal de victimación incrementa la ansiedad o preocupación frente al mismo. Con

\footnotetext{
${ }^{14}$.- Nota del traductor: Nótese, sin embargo, que en Cataluña aunque la incidencia de estas formas delictivas es baja si la comparamos al resto de los países, los sentimientos de inseguridad respecto a la misma no lo son. En otras palabras, no parece existir un claro balance entre los riesgos reales de victimación y las percepciones subjetivas del riesgo de victimación.
}

Revista Española de Criminología

REIC NI02-03 http://www.criminologia.net 
relación al robo en casa hay que indicar que la tendencia ha variado a lo largo de los años, creciendo en general entre 1989 y 1992 y descendiendo después.

\subsection{Sentimientos de seguridad en las calles y en el hogar}

Como una medida adicional acerca de los sentimientos de seguridad de las personas, la EIVD ha preguntado a todos los sujetos la cuestión: “¿Cómo se siente de seguro cuando camina solo por su barrio después de que anochezca?, ¿Se siente muy seguro, medianamente seguro, un poco seguro o muy inseguro?"

Esta cuestión típicamente ha venido a ofrecer una imagen diferente del "miedo al delito" que la obtenidas con cuestiones que preguntan sobre la percepción del riesgo. Típicamente, las mujeres y las personas mayores aparecen como las más temerosas en esta cuestión de "seguridad en las calles". Esto puede deberse a que para algunas personas la posibilidad de estar fuera después del anochecer evoca preocupación sobre un mayor abanico de accidentes y contratiempos (por ejemplo, accidentes pero tambien delitos). La cuestión es también hipotética para aquéllos que rara vez están solos después del anochecer -aunque se instruyó a los entrevistadores/as para preguntar "cómo de seguro se sentiría..." en tales circunstancias. Para las comparaciones a través de países, sin embargo, exactamente lo que mide la cuestión de "seguridad en la calle" es secundario en tanto que es probable que sea interpretado de un modo parecido. Los resultados de sentimientos de seguridad en las calles y en el hogar están en la Figura 6. 


\section{Figura 6. Respecto a estar solo fuera o dentro del hogar después del anochecer}

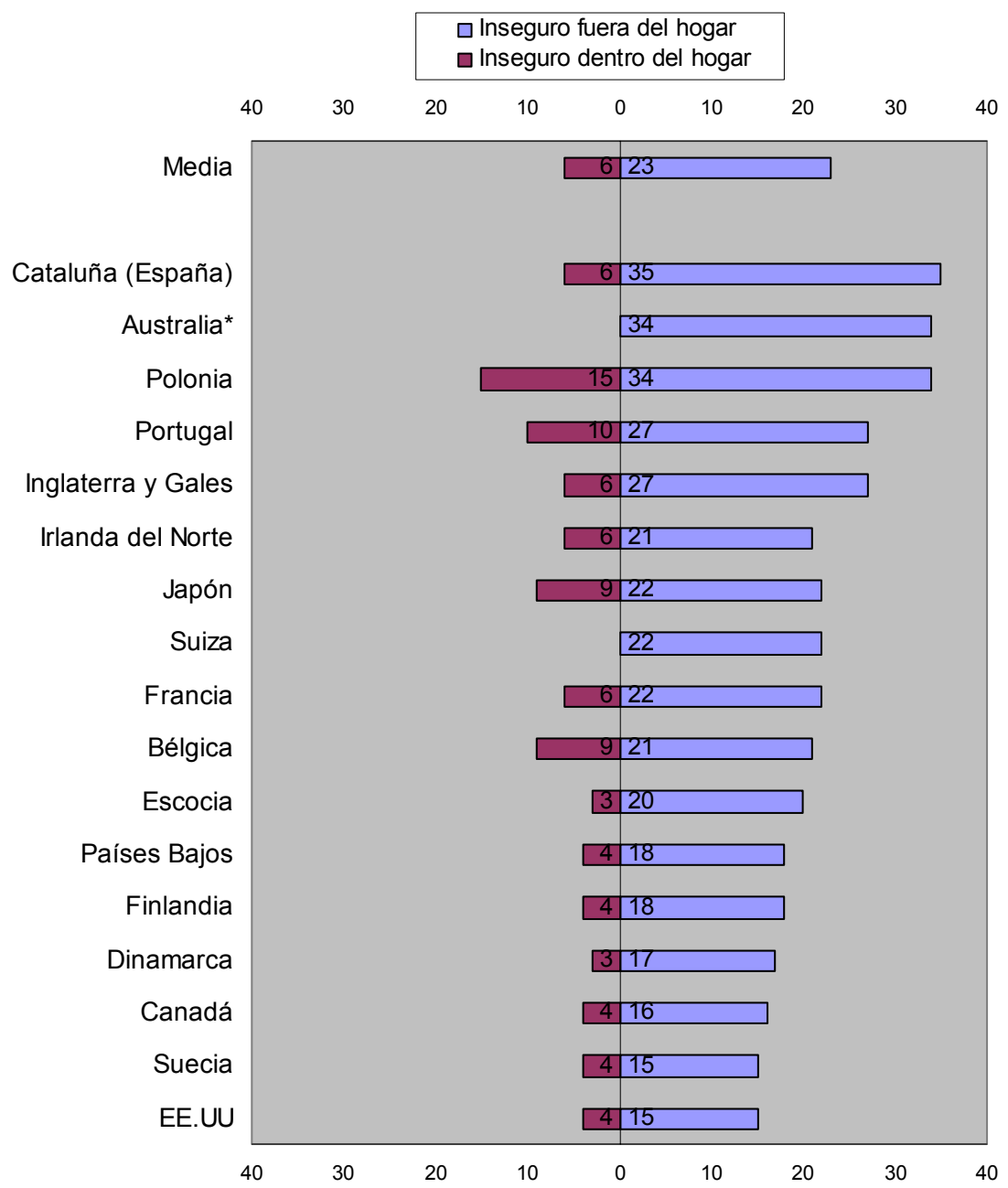

* No se dispone de los resultados de seguridad en el hogar de Australia y Suiza.

Por término medio menos de una cuarta parte se sintieron muy inseguros o un poco inseguros. Aquéllos en Cataluña ${ }^{15}$, Australia y Polonia estuvieron más preocupados por estar fuera solos de noche: sobre una tercera parte se sintieron muy inseguros o un poco inseguros. Los próximos más temerosos fueron aquéllos procedentes de Portugal, Inglaterra y Gales. Por contraste, los sentimientos de inseguridad en las calles de noche fueron más bajos en EEUU y Suecia, pero hubo varios países con otras cifras ligeramente más altas. A lo largo del tiempo, los mayores cambios se han producido en Canadá, los EEUU y Escocia, donde los residentes han llegado a ser menos temerosos de lo que ellos fueron previamente. Por contraste, Suiza se ha movido desde una posición baja a una relativamente alta. En resumen, ha habido

\footnotetext{
${ }^{15}$-- Nota del traductor: Nótese de nuevo como esto ofrece un retrato de una situación en Cataluña donde existe sentimientos de inseguridad que no se corresponden con los niveles objetivos de riesgo.

Revista Española de Criminología

REIC NI02-03 http://www.criminologia.net
} 
una pequeña disminución respecto a la seguridad en las calles basada en una comparación de seis países en tres pases y de once países en los dos más recientes ${ }^{16}$.

En la EIVD de 2000 se introdujo una nueva pregunta sobre sentimientos de seguridad en casa al quedarse solo después del anochecer: “¿Cómo de seguro se siente cuando usted está en casa solo después del anochecer?, ¿Se siente muy seguro, medianamente seguro, un poco inseguro o muy inseguro?".

Una proporción mucho más pequeña de personas se siente insegura en el hogar después del anochecer (6\%) en comparación con las que se sienten así en las calles (23\%) (ver Figura 6). Aquéllas en Polonia se sienten más inseguros en casa (el 15\% se sienten un poco o muy inseguros), seguidos por Portugal, Japón y Bélgica (del 9\% al $10 \%)$.

\subsection{Precauciones de seguridad}

Desde la EIVD de 1992, ha existido una batería suficientemente consistente de preguntas relativas a las medidas tomadas contra los delitos contra la propiedad, en particular el robo en el hogar. A causa de las diferencias entre paises nosotros nos centramos en dos ítems: si se había instalado una alarma anti-robo y si se había instalado una puerta blindada. La Figura 7 muestra que el treinta y cuatro por cierto de los domicilios en Inglaterra y Gales informaron que tenían una alarma anti-robo ${ }^{17}$. Hubo también niveles superiores a la media de propiedad de alarmas en Australia, Escocia, EEUU, Canadá y Bélgica. La propiedad de alarmas fue muy baja en Polonia, Japón y Finlandia. Por término medio, el cuarenta y cuatro por ciento de los domicilios dijeron que tenían puertas blindadas. En general, los países con un nivel relativamente alto de hogares con alarma anti-robo también se clasificaron en un nivel relativamente alto respecto a puertas blindadas. Sin embargo, los Países Bajos en particular estuvieron fuera de esta línea, con la mayor proporción de sujetos que poseían puertas blindadas, pero un término medio inferior de sujetos que poseían en sus hogares alarmas. Ha de observarse que hay una estabilidad razonable en las cifras de los pases de la EIVD en

\footnotetext{
${ }^{16}$.- La proporción media de aquéllos que se sintieron muy o un poco inseguros en los seis países que participaron en los tres pases fue del $25 \%$ en 1992 , $23 \%$ en 1996 y $21 \%$ en 2000 . La media para los once países tanto para la EIVD de 1996 como la de 2000 fue del 23\% en 1996 y del $21 \%$ en 2000.

${ }^{17}$.- Este porcentaje es más alto que el $26 \%$ obtenido en la Encuesta del Delito Británica (British Crime Survey) en el año 2000 (Kershaw et al., 2000).

Revista Española de Criminología

REIC NI02-03 http://www.criminologia.net
} 
cuanto a los niveles de protección en los diferentes países. No obstante, las cifras de protección absoluta se han incrementado en la mayoría de los países ${ }^{18}$.

Figura 7. Porcentaje de hogares con alarmas anti-robo y puertas blindadas

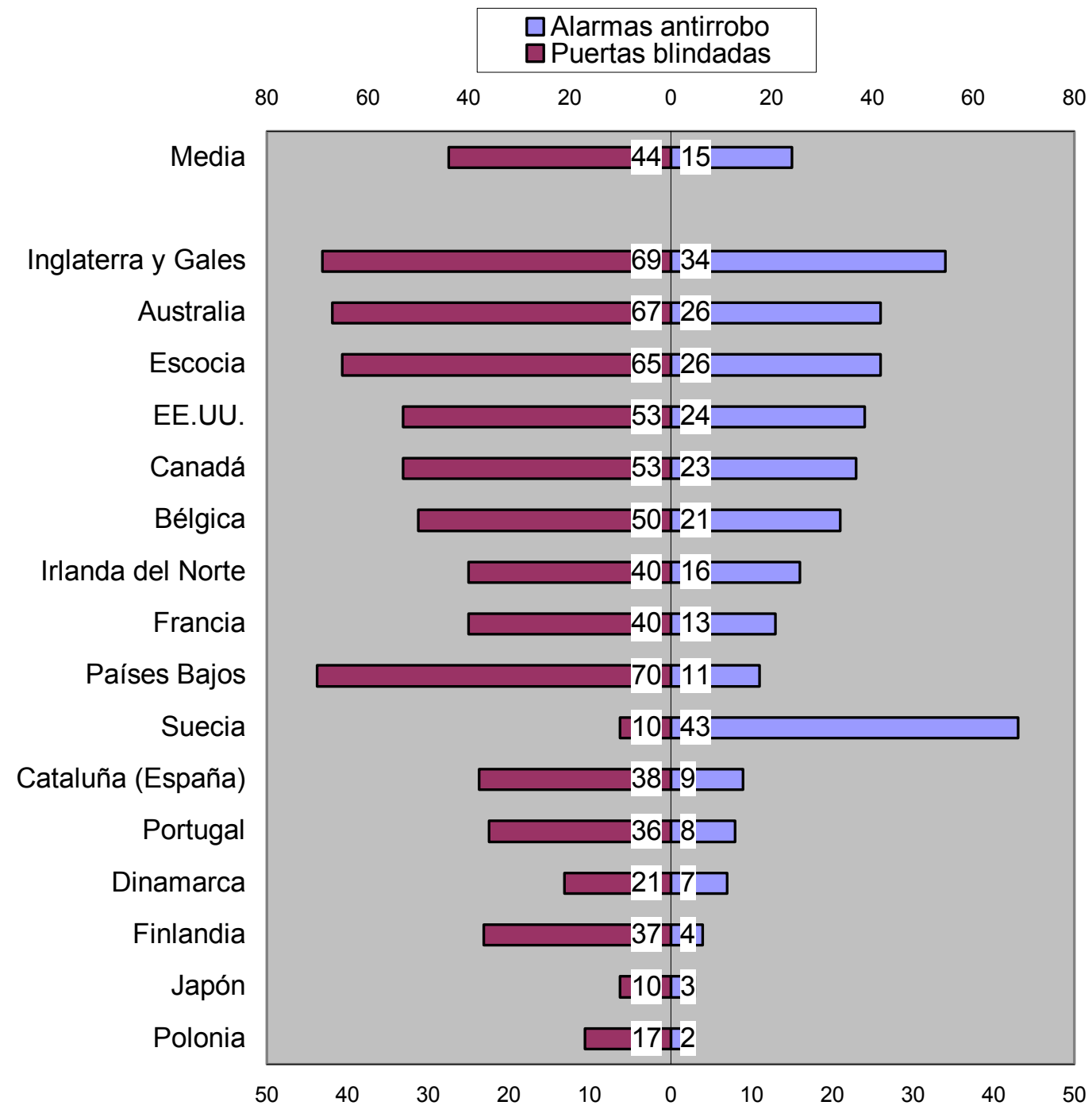

\section{Conclusiones}

Este artículo ha expuesto los principales resultados de la más reciente EIVD en diecisiete países industrializados. Se discutieron temas como los porcentajes de victimación, la gravedad de los delitos sufridos y las principales tendencias durante los últimos años. Además se prestó atención a las diferencias entre países considerando el

\footnotetext{
${ }^{18}$.- Nota del traductor: Nótese que en España, a pesar de que algunos han mostrado cierta alarma por la expansión del mercado de seguridad privada, lo cierto es que estas cifras reflejan claramente que a las firmas de seguridad aun les queda considerable terreno por cubrir y que, si las mismas se pueden emplear como un indicador de su penetración, esta claro que en Cataluña, este tipo de medidas de prevención privadas es relativamente bajo si lo comparamos al del resto de países industrializados.
}

Revista Española de Criminología

REIC NI02-03 http://www.criminologia.net 
comportamiento de denuncia de las víctimas y las opiniones de personas en general sobre la actuación de la Policía.

Los principales resultados se pueden resumir del siguiente modo:

- Cuando observamos en conjunto las cifras de victimación, los países se dividen en tres bandas. Australia, Inglaterra y Gales, los Países Bajos y Suecia muestran las cifras más altas (más del 24\% (de los entrevistados) fueron víctimas de un delito en 1999). Estos países son seguidos por Canadá, Escocia, Dinamarca, Polonia, Bélgica, Francia y EEUU con niveles intermedios (20\%-24\%) y Finlandia, Cataluña (España), Suiza, Portugal, Japón e Irlanda del Norte con los niveles más bajos (menos del 20\%).

- Considerando las tendencias del delito, la EIVD sugiere que el delito ascendió entre 1988 y 1991, no mostró excesivo cambio en 1995 y luego descendió en 1999.

Este es el modelo dominante en muchos países.

- De los once delitos examinados, el robo de coche en el que el coche no se recobra fue considerado el más grave. La clasificación de delitos en términos de gravedad fue muy parecida en todos los países. Esto indica un algo grado de consenso respecto a la importancia de los delitos convencionales.

- Sólo la mitad de los delitos sufridos en los países industrializados se denunciaron a la Policía, así hay una considerable "cifra negra" de delitos no recogidos en las estadísticas policiales. Como los delitos más graves se denuncian más frecuentemente a la Policía, las cifras policiales reflejarán mejor este tipo de delitos.

- La mayoría de las víctimas se sintieron satisfechas con el trato recibido por la Policía cuando ellas fueron a denunciar el delito; la cifra fue algo más baja para los delitos contra las personas que para los delitos contra la propiedad. Esto puede deberse a la mayor dificultad de las víctimas para denunciar cuando éstas presencian el delito, o el hecho de que denunciar delitos contra la propiedad tiene a menudo más que ver con consideraciones del seguro que con la expectativa de lo que la Policía haría o podría hacer.

- Hubo un aumento en los servicios especializados para víctimas desde principios de los años ochenta y pases anteriores de la EIVD han indicado una proporción creciente de víctimas recibiendo ayuda. 
- Cuando se preguntó cómo de seguras se sentían las personas caminando solas por su barrio después del anochecer, menos de una cuarta parte se sintieron muy o un poco inseguros. Una proporción mucho menor se sintieron inseguros en su hogar.

Todos estos resultados plantean muchas cuestiones sobre las causas de las diferencias entre países y sobre cómo explicar las tendencias dentro de cada país. Otros artículos (en este libro $)^{19}$ intentarán responder a algunos de estos interrogantes. Las preguntas que quedan tendrán que responderse en estudios futuros, en los que las EIVD serán, indudablemente, de gran ayuda. ${ }^{20}$

\section{Esther Bouten}

\section{Heike Goudriaan}

\section{Paul Nieuwbeerta}

iD https://orcid.org/0000-0001-5134-3175

19 .- Nota del traductor: Ver NCSR, Nieuwbeerta, P. (ed.), (2002) Crime Victimization in comparative perspective. Results from the International Crime Victims Survey, 1989-2000. Den Haag: Boom Juridische uitgevers.

20.- Nota del traductor: En cuanto a Cataluña las conclusiones más importantes son las siguientes:

- Cataluña pertenece al grupo de países con niveles bajos de victimación

- A pesar de ello los sentimientos de inseguridad ciudadana y las percepciones de riesgo son muy elevados y no se encuentran en consonancia con los niveles objetivos de riesgo lo que sugiere el uso de estrategias policiales para la reducción del miedo y discursos políticos menos alarmistas

- Aunque las victimas que denuncian delitos a la policía se encuentran generalmente satisfechas con la atención recibida, Cataluña se ubica entre los países en los que existe un mayor escepticismo sobre la eficacia de la labor policial, lo que se refleja en una tasa de denuncias de las mas bajas, lo que sugiere la adopción de adecuadas estrategias de relaciones publicas por parte de la policía

- Cataluña presenta niveles muy bajos de medidas privadas de seguridad del hogar, en comparación con otros países industrializados, y también niveles muy bajos de asistencia a la víctima del delito, lo que sugiere que es preciso fomentar medidas en este terreno

Revista Española de Criminología

REIC NI02-03 http://www.criminologia.net

https://doi.org/10.46381/reic.v1i0.8 